\title{
Spectral stability of Markov systems
}

\author{
P. Duarte ${ }^{1}$, M. J. Torres ${ }^{2}$
}

1 CMAF, Faculdade de Ciências da Universidade de Lisboa, Campo Grande, Edifício CS, 1749-016 Lisboa, Portugal (e-mail: pduarte@ptmat.fc.ul.pt)1

${ }^{2}$ CMAT, Departamento de Matemática, Universidade do Minho, Campus de Gualtar, 4700057 Braga, Portugal (e-mail: jtorres@math.uminho.pt) ${ }^{2}$

This is an author-created, un-copyedited version of an article accepted for publication in Nonlinearity. IOP Publishing Ltd is not responsible for any errors or omissions in this version of the manuscript or any version derived from it. The definitive publisher authenticated version is available online at http://dx.doi.org/10.1088/0951-7715/21/3/001.

Abstract For a class of semigroups of stochastic dynamical systems, $x \mapsto P_{x}$, where $x$ denotes a state and $P_{x}$ the state probability transition, we relate its spectral stability with the combinatorial stability of the underlying non-deterministic dynamics, associated to the point-set map $x \mapsto \operatorname{supp}\left(P_{x}\right)$.

\section{Introduction}

Uniformly hyperbolic (Axiom A) systems were introduced by Smale, see [Sm], in the early sixties, and have been widely studied both from the topological and the ergodic view-point, see e.g. [S] and references therein. Smooth deterministic uniformly hyperbolic systems $f: X \rightarrow X$ admit a precise description of their dynamics: the spectral

\footnotetext{
${ }^{1}$ The first author was supported by Research Center of Mathematics and Fundamental Applications of the University of Lisbon through the FCT Pluriannual Funding Program.

${ }^{2}$ The second author was supported by the Research Centre of Mathematics of the University of Minho through the FCT Pluriannual Funding Program.
} 
decomposition theorem states that there is a decomposition of the non-wandering set $\Omega(f)$ into a finite number of hyperbolic basic sets which are permuted by $f$. The dynamics of $f$ partially orders the basic set components of $\Omega(f)$, the minimal, or final, elements being the attractors of $f$. As the name indicates this decomposition relates with the spectral decomposition of the linear operator which describes the action of $f$ on the tangent vector fields to $X$. In the seventies, the ergodic theory of uniformly hyperbolic systems was established by the work of Sinai, Ruelle and Bowen. See [Si1], $[\mathrm{Ru}]$, [BR], [Bo]. For these dynamical systems each attractor has a physical measure, whose basin of attraction cover almost every point is state space. Physical measures were first constructed by Sinai [Si1] for Anosov diffeomorphisms. This was extended by Ruelle $[\mathrm{Ru}]$ for general hyperbolic (Axiom A) diffeomorphisms, and by BowenRuelle [BR] for Axiom A flows. A physical measure of an attractor is the one that describes the system time average for a set of initial states with positive volume. The set of all such initial states is called the physical measure basin of attraction. A measure on an attractor is said to be stochastically stable, concept introduced by Kolmogorov and Sinai, if it is stable under small stochastic perturbations of the deterministic system. More precisely, introducing a random noise, the limit measures of the random perturbations approach the attractor's physical measure as the noise level tends to zero. See, e.g., [V1]. The key idea of introducing a random noise in a deterministic system, and then looking at the limit measures as the noise level tends to zero, goes back to Kolmogorov. See [Si2]. Kolmogorov expressed the idea that zero-noise limits represent measures that yield a certain "physical" insight of the system's behaviour. See [BDV], [Y2]. The effects of small random errors on the asymptotic distribution of points in the basin of a hyperbolic attractor were considered, for different perturbation schemes, by Kifer and Young. They established the stability of uniformly hyperbolic attractors, for different models of random perturbations. See [K1], [K3], [Y1]. See also [K4] and [V1]. But, beyond the Axiom A setting, the problem of existence and finiteness of physical measures and their stability, remains as a major purpose in dynamical systems. An open conjecture by Palis $[\mathrm{P}]$ is that every dynamical system can be approximated by one with finitely many attractors, each having a stochastically stable physical measure, whose basins of attraction cover almost every point in state space. See, e.g., [V2]. A good comprehension of which dynamical systems admit physical measures was not yet achieved, but some work has been done. See [BDV], [V2], [Y2] for surveys on much of the progress already made. See also [Li] for a recent survey on random dynamical systems. Under very mild conditions, a random noise can have a powerful simplifying effect on the complexity of the dynamics of a deterministic system. Namely, under arbitrary small random perturbations any deterministic system has 
finitely many attractors (see, for instance, $[\mathrm{A}]$ ). The spectrum of the Perron-Frobenius operator, which reflects the action of dynamics upon measures, may also be simplified. In general, the spectrum of this linear operator can be complex, but when we add a random noise this usually makes the operator compact or weakly compact with pure point spectrum. A compact operator can be, from the spectral point of view, well approximated by finite-dimension operators. Thus random perturbations of a deterministic system may, just as well, be considered on finite (discrete) approximations of state space. Finite state Markov chains can be viewed as stochastic, or random dynamical systems on a finite state space. One may think that these dynamical systems are what we actually see when running computer simulations of deterministic dynamical systems. Each such dynamical system is specified by a stochastic matrix with the state probability transitions. The Perron operator of this finite state system is the stochastic matrix. The Markov chain also determines an oriented graph, encapsulating some qualitative aspects of the system behaviour. The theory of finite state Markov chains establishes a correspondence between spectral properties of the stochastic matrix on one side, and combinatorial properties of the corresponding graph on the other hand. See, e.g., [B], [D]. This theory can be nicely extended into a theory for continuous, or lower semi-continuous, random dynamical systems of Markov type on a compact manifold, which is the main purpose of this work. In a previous article [DT] we have developed such a theory in its topological and combinatorial aspects, which we now summarise.

\section{Combinatorial Stability of Open Maps}

From now on $X$ will denote a compact Riemannian manifold and $m$ will be the corresponding normalized Riemannian volume on $X$, i.e. $m(X)=1$. We denote by $\mathcal{O}(X)$ the space of all multi-valued $\operatorname{maps}^{3} \varphi: X \rightarrow \mathcal{P}(X)$ whose graph graph $\varphi=$ $\{(x, y) \in X \times X: y \in \varphi(x)\}$ is an open set in $X \times X$, and such that $\varphi(x)$ is non-empty and connected for every $x \in X$. Such systems will be referred as open maps. Given two open maps $\varphi, \psi: X \rightarrow \mathcal{P}(X)$ the composition $\varphi \circ \psi: X \rightarrow \mathcal{P}(X)$ of $\varphi$ and $\psi$ at $x$ is defined to be $(\varphi \circ \psi)(x):=\varphi(\psi(x))=\cup_{y \in \psi(x)} \varphi(y)$. With this composition, $\mathcal{O}(X)$ is a semigroup. We think of open maps on $X$ as non-deterministic dynamical systems with state space $X$. For an open map $\varphi: X \rightarrow \mathcal{P}(X)$ and a subset $A \subseteq X$

\footnotetext{
${ }^{3}$ A multi-valued or point-set map $\varphi: X \rightarrow \mathcal{P}(Y)$ is one that to each point $x \in X$ associates some set $\varphi(x) \subseteq Y$. See $[\mathrm{AF}]$ for an overview on multi-valued analysis.
} 
the image $\varphi(A) \in \mathcal{P}(X)$ is defined by $\varphi(A)=\cup_{x \in A} \varphi(x)$, and we say that $A \subseteq X$ is $\varphi$-invariant when $\varphi(A) \subseteq A$. Let us now briefly recall the main dynamical concepts for open maps. Given $\varphi \in \mathcal{O}(X)$, a sequence $x_{0}, x_{1}, \cdots, x_{n}$ such that $x_{i} \in \varphi\left(x_{i-1}\right)$ for all $i=1, \cdots, n$ is called a $\varphi$-orbit, and we say that $x_{n}$ is a $\varphi$-iterate of $x_{0}$. If for every $\epsilon>0, y$ is a $\varphi_{\epsilon}^{*}$-iterate of $x$, where $\varphi_{\epsilon}^{*}$ is the open map whose graph is an $\epsilon$-radius ball of graph $(\varphi)$, we say that $y$ is a $\varphi$-pseudo-iterate of $x$. The recurrent and chainrecurrent sets of $\varphi$ are defined respectively by $\Omega(\varphi)=\{x \in X: x$ is a $\varphi$-iterate of $x\}$ and $R(\varphi)=\{x \in X: x$ is a $\varphi$-pseudo-iterate of $x\}$. Both these sets split into equivalence classes, each class being formed by states which are accessible from each other. The set of all these classes is then partially ordered by the dynamics of $\varphi$. At the bottom of this hierarchy are two special limit sets: the final recurrent and the final chain-recurrent sets, denoted respectively by $\Omega_{\text {final }}(\varphi)$ and $R_{\text {final }}(\varphi)$, of all states $x \in \Omega(\varphi)(x \in R(\varphi))$ such that every iterate (pseudo-iterate) of $x$ still has some iterate (pseudo-iterate) which comes back to $x$. These limit sets contain all the asymptotic dynamical behaviour of $\varphi$. They both decompose into a finite number of equivalence classes, called respectively $\Omega$-final and $R$-final classes. We denote by $\Lambda_{\text {final }}^{\Omega}(\varphi)$ respectively $\Lambda_{\text {final }}^{R}(\varphi)$ the set of all equivalence classes of the limit sets $\Omega_{\text {final }}(\varphi)$ and $R_{\text {final }}(\varphi)$. Each $\Omega$-final and $R$-final class decomposes into a finite number of connected pieces, called respectively $\Omega$-final and $R$-final components, which are permuted by $\varphi$. See Theorems 5.1 and 5.2 of [DT]. The restriction of $\varphi$ to each of these pieces is in some sense irreducible. We call period of a final class to the number of its connected components. The period of a connected component is the period of its class. We denote by $\Sigma_{\text {final }}^{\Omega}(\varphi)$ respectively $\Sigma_{\text {final }}^{R}(\varphi)$ the set of connected pieces of the limit sets $\Omega_{\text {final }}(\varphi)$ and $R_{\text {final }}(\varphi)$. Thus, each open map $\varphi \in \mathcal{O}(X)$ induces a permutation $\pi_{\varphi}$ on the set $\Sigma_{\text {final }}^{\Omega}(\varphi)$ of $\Omega$-final components. Given $\varphi, \psi \in \mathcal{O}(X)$, we say that $\varphi$ is combinatorially equivalent to $\psi$ if and only if the permutations $\pi_{\varphi}$ and $\pi_{\psi}$ are conjugated, that is, there is a bijective map $h: \Sigma_{\text {final }}^{\Omega}(\varphi) \rightarrow \Sigma_{\text {final }}^{\Omega}(\psi)$ such that $\pi_{\psi} \circ h=h \circ \pi_{\varphi}$. Next we topologize the sub-semigroups $\mathcal{O}_{1}$ of $\mathcal{O}(X)$ in order to prove that under general conditions generic elements of $\mathcal{O}_{1}$ are combinatorially stable. To be precise, if the semigroup $\mathcal{O}_{1}$ has some topology we say that $\varphi \in \mathcal{O}_{1}$ is combinatorially stable if and only if $\varphi$ is combinatorially equivalent to every $\psi$ in some neighborhood of $\varphi$. Because there are several natural non-equivalent ways of endowing $\mathcal{O}(X)$, and its sub-semigroups, with some topology we give the following abstract definition:

Definition 2.1. We say that $\mathcal{O}_{1}$ is a topological semigroup of open maps if

(1) the Hausdorff distance between open map graphs is continuous;

(2) for each $\varphi \in \mathcal{O}_{1}$, there is a family of open maps $\left\{\tilde{\varphi}_{\epsilon}\right\}_{\epsilon>0}$ in $\mathcal{O}_{1}$ such that 
(a) $\overline{\operatorname{graph}(\varphi)}=\bigcap_{\epsilon>0} \operatorname{graph}\left(\tilde{\varphi}_{\epsilon}\right)$;

(b) for all $\epsilon_{1}, \epsilon_{2}$, if $\epsilon_{1}>\epsilon_{2}>0$ then $\overline{\operatorname{graph}\left(\tilde{\varphi}_{\epsilon_{2}}\right)} \subseteq \operatorname{graph}\left(\tilde{\varphi}_{\epsilon_{1}}\right)$; and

(c) $\lim _{\epsilon \rightarrow 0^{+}} \tilde{\varphi}_{\epsilon}=\varphi$ w.r.t. the topology of $\mathcal{O}_{1}$.

(3) given $\epsilon>0$, an integer $N$, and non-empty open subsets $U, V \subseteq X$ such that $\overline{U \times V} \subseteq \operatorname{graph}\left(\varphi^{N}\right)$, there is a neighborhood $\mathcal{N}$ of $\varphi$ in $\mathcal{O}_{1}$ such that for all $\psi \in \mathcal{N}$ and $x \in \bar{U}, \quad m\left(V \backslash \widehat{\psi^{N}}(x)\right)<\epsilon$, where $\widehat{\psi}$ denotes the regularization of $\psi$.

We say that an open set $U$ is regular if $U=(\bar{U})^{\circ}$. In general, the set $\widehat{U}:=(\bar{U})^{\circ}$, the interior of the closure of $U$, is called the regularization of $U$. Similarly, for open maps $\psi \in \mathcal{O}(X)$ the open map $\widehat{\psi}$ defined by $\operatorname{graph}(\widehat{\psi})=\widehat{\operatorname{graph}(\psi)}$ is called the regularization of $\psi$. Since open maps are open subsets of $X \times X$, in [DT] we consider, on $\mathcal{O}(X)$ and its sub-semigroups, topologies induced from general (topological) spaces of open sets. See $[\mathrm{N}]$ for an overview on topological spaces of sets. Condition (2) above is an outer continuity assumption that says every open map $\varphi$ can be well approximated from above within the topology. Condition (3) expresses a kind of inner, or lower, continuity. With this concept we prove the following theorems. See theorems 5.3 and 5.4 of $[\mathrm{DT}]$. Assume $\mathcal{O}_{1} \subseteq \mathcal{O}(X)$ is any topological semigroup of open maps. Then:

Theorem 1 An open map $\varphi \in \mathcal{O}_{1}$ is combinatorially stable in $\mathcal{O}_{1}$ if and only if $\varphi$ induces the same permutation on $\Sigma_{\text {final }}^{\Omega}(\varphi)$ and $\Sigma_{\text {final }}^{R}(\varphi)$. This in turn is equivalent to $\left|\Lambda_{\text {final }}^{\Omega}(\varphi)\right|=\left|\Lambda_{\text {final }}^{R}(\varphi)\right|$ and $\left|\Sigma_{\text {final }}^{\Omega}(\varphi)\right|=\left|\Sigma_{\text {final }}^{R}(\varphi)\right|$.

This shows that the combinatorial stability of an open map $\varphi \in \mathcal{O}_{1}$ is independent of the topology in $\mathcal{O}_{1}$.

Theorem 2 The set of $\mathcal{O}_{1}$-combinatorially stable maps is open and dense in $\mathcal{O}_{1}$.

\section{Markov Systems}

We denote by $\mathcal{M}_{\text {prob }}(X)$ the space of all Borel probability measures on the compact manifold $X$. This is a subset of the Banach space $\mathcal{M}(X)$ of all finite Borel real measures on $X$, with the usual total variation norm $\|\mu\| . \mathcal{M}(X)$ is the dual of the Banach space of continuous real-valued functions on $X$, denoted here by $C^{0}(X)$, endowed with the uniform proximity norm $\|\cdot\|_{\infty}$. The space $\mathcal{M}_{\text {prob }}(X)$ is a compact and convex subset of $\mathcal{M}(X)$ with respect to the weak-* topology, which is the weak topology of 
$\mathcal{M}(X)$ as dual of $C^{0}(X)$. We will call here Markov system to any weak-* continuous mapping $p: X \rightarrow \mathcal{M}_{\text {prob }}(X)$. The probability measure $p(x)=p_{x}$ is referred as the transition probability at state $x \in X$. We denote by $\mathcal{M S}(X)$ the set of all Markov systems. A Markov system $p: X \rightarrow \mathcal{M}_{\text {prob }}(X)$ will also be referred as a stochastic dynamical system. A Markov system is called deterministic if for some continuous mapping $f: X \rightarrow X$, we have $p(x)=\delta_{f(x)}$ for every $x \in X$, where $\delta_{f(x)}$ denotes the Dirac measure sitting at the point $f(x)$. The Perron-Frobenius operator of a Markov system $p: X \rightarrow \mathcal{M}_{\text {prob }}(X)$ is the linear operator $\mathcal{L}_{p}: \mathcal{M}(X) \rightarrow \mathcal{M}(X)$, defined by

$$
\mathcal{L}_{p}(\mu)=\int_{X} p(x) d \mu(x), \quad \text { for every } \mu \in \mathcal{M}(X) .
$$

The integral of the measure-valued function $p$ is well defined, in a sense that can be found, for instance, in $[\mathrm{Ru}]$. The adjoint operator $\mathcal{L}_{p}^{*}: C^{0}(X) \rightarrow C^{0}(X)$, is given by

$$
\mathcal{L}_{p}^{*}(\psi)(x)=\int_{X} \psi(y) d p_{x}(y), \quad \text { for every } \psi \in C^{0}(X) .
$$

Both $\mathcal{L}_{p}$ and $\mathcal{L}_{p}^{*}$ are bounded linear operators with norms less or equal than 1 . The convolution of two Markov systems $p, q \in \mathcal{M S}(X)$ is $p * q: X \rightarrow \mathcal{M}_{\text {prob }}(X)$, where

$$
(p * q)(x)=\mathcal{L}_{p}\left(q_{x}\right)=\mathcal{L}_{p}\left(\mathcal{L}_{q}\left(\delta_{x}\right)\right) \quad \text { for every } x \in X .
$$

The space $(\mathcal{M S}(X), *)$ is a semigroup with identity, where the identity is the deterministic Markov system $x \mapsto \delta_{x}$. The map $p \mapsto \mathcal{L}_{p}$ is a semigroup homomorphism taking $\mathcal{M S}(X)$ into the algebra of bounded linear operators on the Banach space $\mathcal{M}(X)$. We will say that a measure $\mu \in \mathcal{M}(X)$ is $p$-invariant when $\mathcal{L}_{p} \mu=\mu$, and that a measurable set $A \subseteq X$ is $p$-invariant when $\mathcal{L}_{p}^{*} \chi_{A}=\chi_{A}$, where $\chi_{A}$ denotes the characteristic function $\chi_{A}(x)=\left\{\begin{array}{lll}1 & \text { if } & x \in A \\ 0 & \text { if } & x \in X-A\end{array}\right.$.

We denote by $L^{1}(X, m)$ the Banach space of $m$-integrable real functions on $X$ with the usual $L^{1}$-norm, $\|h\|_{1}=\int_{X}|h(x)| d m(x)$. This space is isometrically embedded in $\mathcal{M}(X)$ through the inclusion map $L^{1}(X, m) \hookrightarrow \mathcal{M}(X), \quad h \mapsto h m$. We say that a Markov system $p: X \rightarrow \mathcal{M}(X)$ is absolutely continuous with respect to $m$ if $p_{x}=f_{x} m$, with $f_{x} \in L^{1}(X, m)$, for every $x \in X$. Absolutely continuous Markov systems are defined by stochastic transition functions $f: X \times X \rightarrow \mathbb{R}$ such that:

(a) $f(x, y)$ is measurable on $X \times X$,

(b) $f(x, y) \geq 0$, for every $(x, y) \in X \times X$, 
(c) $\int_{X} f(x, y) d m(y)=1$, for every $x \in X$,

(d) the real valued function $X \rightarrow \mathbb{R}, x \mapsto \int f(x, y) \psi(y) d m(y)$, is continuous for every test function $\psi \in C^{0}(X)$.

A function $f: X \times X \rightarrow \mathbb{R}$ satisfying (a), (b), (d) and

(c') $\int_{X} f(x, y) d m(y) \leq 1$, for every $x \in X$,

is called a sub-stochastic transition function.

The subset of all absolutely continuous Markov systems forms a sub-semigroup, without identity, of $\mathcal{M S}(X)$. Given two transition functions $f, g: X \times X \rightarrow \mathbb{R}$, the convoluted Markov system is defined by the usual function convolution

$$
(f * g)(x, z)=\int_{X} f(x, y) g(y, z) d m(y) .
$$

From now on we shall identify each absolutely continuous Markov system with its probability transition function $f: X \times X \rightarrow \mathbb{R}$. Given any such absolutely continuous Markov system $f$, the operator $\mathcal{L}_{f}$ takes $\mathcal{M}(X)$ into $L^{1}(X, m)$ and its restriction to $L^{1}(X, m)$ is given by

$$
\mathcal{L}_{f}(q)(y)=\int_{X} q(x) f(x, y) d m(x) \quad q \in L^{1}(X, m) .
$$

The adjoint action on $L^{\infty}(X, m)$ is given by

$$
\mathcal{L}_{f}^{*}(g)(x)=\int_{X} f(x, y) g(y) d m(y) \quad g \in L^{\infty}(X, m) .
$$

Given a Markov system $p: X \rightarrow \mathcal{M}_{\text {prob }}(X), \sigma\left(\mathcal{L}_{p}\right)$ will denote the spectrum of the Perron-Frobenius operator $\mathcal{L}_{p}$. The spectral radius of $\mathcal{L}_{p}$, i.e. the lowest upper bound for absolute values of elements in $\sigma\left(\mathcal{L}_{f}\right)$, will be denoted by $r\left(\mathcal{L}_{p}\right)$. Of course $r\left(\mathcal{L}_{p}\right)=1$. The discrete spectrum of $\mathcal{L}_{p}$, i.e. the set of all eigenvalues in $\sigma\left(\mathcal{L}_{p}\right)$ that are isolated and have finite multiplicity, will be denoted by $\sigma_{\text {disc }}\left(\mathcal{L}_{p}\right)$. The complement of $\sigma_{\text {disc }}\left(\mathcal{L}_{p}\right)$ in $\sigma\left(\mathcal{L}_{p}\right)$ is called the essential spectrum of $\mathcal{L}_{p}$, and denoted by $\sigma_{\text {ess }}\left(\mathcal{L}_{p}\right)$. The essential spectral radius of $\mathcal{L}_{p}$, i.e. the lowest upper bound for absolute values of elements in $\sigma_{\text {ess }}\left(\mathcal{L}_{f}\right)$, is denoted by $r_{\text {ess }}\left(\mathcal{L}_{p}\right)$. It is well known, see for instance [W], that the Perron operator $\mathcal{L}_{f}$ of any absolutely continuous Markov system $f$ is a weakly 
compact operator. In particular, $r_{\text {ess }}\left(\mathcal{L}_{f}\right)=0$ and, therefore, the spectrum $\sigma\left(\mathcal{L}_{f}\right)$ is at most countable. All spectrum points in $\sigma\left(\mathcal{L}_{f}\right)-\{0\}$ are isolated eigenvalues with finite multiplicity. Given an absolutely continuous Markov system $f$, we can decompose the spectrum of $\mathcal{L}_{f}$ as:

$$
\sigma\left(\mathcal{L}_{f}\right)=\sigma_{0}\left(\mathcal{L}_{f}\right) \cup \sigma_{1}\left(\mathcal{L}_{f}\right)
$$

where $\sigma_{0}\left(\mathcal{L}_{f}\right)=\left\{\lambda \in \sigma\left(\mathcal{L}_{f}\right):|\lambda|<1\right\}$, and $\sigma_{1}\left(\mathcal{L}_{f}\right)=\sigma\left(\mathcal{L}_{f}\right)-\sigma_{0}\left(\mathcal{L}_{f}\right)$. Of course $\sigma_{1}\left(\mathcal{L}_{f}\right)$ is finite while $\sigma_{0}\left(\mathcal{L}_{f}\right)$ is at most countable but closed for the complex plane topology. Consequently, $\sigma_{0}\left(\mathcal{L}_{f}\right)$ and $\sigma_{1}\left(\mathcal{L}_{f}\right)$ are disjoint compact sets and, therefore, there is an associated decomposition of $L^{1}(X, m)$ into two $\mathcal{L}_{f}$-invariant subspaces:

$$
L^{1}(X, m)=E_{0}(f) \oplus E_{1}(f)
$$

where $E_{1}(f)$ has finite dimension. We shall denote by $r_{\text {int }}\left(\mathcal{L}_{f}\right)$ the interior spectral radius of $\mathcal{L}_{f}$, i.e. the lowest upper bound of all absolute values of elements in $\sigma_{0}\left(\mathcal{L}_{f}\right)$.

Given any absolutely continuous Markov system $f$ a sequence $x_{0}, x_{1}, \cdots, x_{n}$ such that $f\left(x_{i-1}, x_{i}\right)>0$ for all $i=1, \cdots, n$ is called an $f$-orbit, and we say that $x_{n}$ is an $f$-iterate of $x_{0}$. An absolutely continuous Markov system is called irreducible if for almost all points $x, y \in X$ there is some $n \in \mathbb{N}$ such that the probability transition density from $x$ to $y$ in $n$ iterates is positive, i.e. $f^{n}(x, y)>0$. A recurrence time is any integer $n \in \mathbb{N}$ such that $f^{n}(x, x)>0$ with positive probability. Given an absolutely continuous irreducible Markov system $f$ the greatest common divisor $d$ of all recurrence times $n \in \mathbb{N}$ is called the period of $f$. An irreducible Markov system $f$ is called acyclic if it has period one. The state space $X$ of an irreducible Markov system $f$ of period $d$

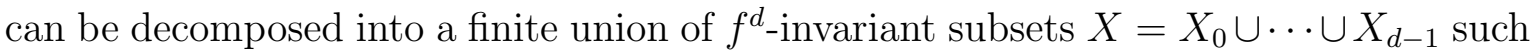
that each restriction $\left(f^{d}\right)_{X_{i}}: X_{i} \times X_{i} \rightarrow \mathbb{R}$, is an irreducible acyclic Markov system on $X_{i}$.

We shall denote by $f_{R}$ the restriction to $R \times R$ of a given function $f: X \times X \rightarrow \mathbb{R}$, for any subset $R \subseteq X$. If $f$ is stochastic transition function then:

1. $f_{R}$ is a sub-stochastic transition function.

2. $f_{R}$ is a stochastic transition function $\Leftrightarrow R$ is $f$-invariant. 


\section{Statement of Results}

Let $\mathcal{H}(X)$ be the set of all absolutely continuous Markov systems (i.e. probability transition functions) $f: X \times X \rightarrow \mathbb{R}$ satisfying the following extra conditions:

(1) $f$ is bounded on $X \times X$,

(2) $f$ is lower semi-continuous on $X \times X$,

(3) for each $x \in X$, the open set $\varphi_{f}(x)=\{y \in X: f(x, y)>0\}$ is connected.

The space $\mathcal{H}(X)$ is a convolution sub-semigroup of $\mathcal{M S}(X)$. Item (2) in the definition of $\mathcal{H}(X)$ ensures that $\varphi_{f} \in \mathcal{O}(X)$. Thus, this semigroup carries a natural homomorphism $\varphi: \mathcal{H}(X) \rightarrow \mathcal{O}(X)$. Our motivation to consider this space was the fact that it could be used, in some future work, to address the subtle concept of stochastic stability for continuous deterministic dynamical systems, since $\mathcal{H}(X)$ is suitable to consider random perturbations of such deterministic systems. The main goal of this work is to relate, for generic systems $f \in \mathcal{H}(X)$, the combinatorial stability of $\varphi_{f}$ with the spectral stability of $f$, defined below. The novelty here with respect to finite state Markov chain theory is that in this context, because we are dealing with continuous systems, it makes sense defining stability: combinatorial stability or spectral stability. Assume $\mathcal{H}_{1} \subseteq \mathcal{H}(X)$ is sub-semigroup endowed with some topology.

Definition 4.1. We say that $f \in \mathcal{H}(X)$ is spectrally stable in $\mathcal{H}_{1}$ if and only if there is a neighborhood $\mathcal{U}$ of $f$ in $\mathcal{H}_{1}$ and there is $0<k<1$ such that for all $g \in \mathcal{U}$ :

(1) There is a linear map $h_{g}: E_{1}(f) \rightarrow E_{1}(g)$ that conjugates $\left.\mathcal{L}_{f}\right|_{E_{1}(f)}$ to $\left.\mathcal{L}_{g}\right|_{E_{1}(g)}$.

(2) The map $h_{g}$ depends continuously on $f$ w.r.t. the topology in $\mathcal{H}_{1}$, in the sense that for any $\varphi \in C^{0}(X), \lambda_{\varphi} \circ h_{g}$ converges to $\lambda_{\varphi}$ as $g$ tends to $f$ in $\mathcal{H}_{1}$, where $\lambda_{\varphi}: L^{1}(X, m) \rightarrow \mathbb{R}$ is defined by $\lambda_{\varphi}(\mu)=\int \varphi d \mu$.

(3) $\sigma_{0}\left(\mathcal{L}_{g}\right) \cap\{\lambda \in \mathbb{C}: k<|\lambda|<1\}=\varnothing$.

We note that item (2) above is equivalent to say that the invariant measures of $\mathcal{L}_{f}$ vary continuously with $f$ w.r.t. the weak-* topology. The fixed points of this linear operator are precisely the system invariant measures. The spectral stability of $f$ relates with the fact that no eigenvalues can enter, or leave, the unit circle. Given $\varphi, \psi \in \mathcal{O}(X)$, we will write $\varphi \prec \psi$ to mean that $\overline{\operatorname{graph}(\varphi)} \subseteq \operatorname{graph}(\psi)$. Consider now any sub-semigroup of Markov systems $\mathcal{H}_{1} \subseteq \mathcal{H}(X)$, endowed with some topology. 
Definition 4.2. We say that $\mathcal{H}_{1}$ is a topological semigroup of Markov systems over a topological semigroup of open maps $\mathcal{O}_{1}$ if and only if for any $f \in \mathcal{H}_{1}$ :

(1) $\varphi_{f} \in \mathcal{O}_{1}$;

(2) The map $f \mapsto \varphi_{f}$ is continuous for the topology of $\mathcal{O}_{1}$;

(3) $\mathcal{H}_{1}$ admits outer approximations in the sense that given $f \in \mathcal{H}_{1}$, for every neighborhood $\mathcal{N}$ of $f$ in $\mathcal{H}_{1}$ there is $g \in \mathcal{N}$ such that $\varphi_{f} \prec \varphi_{g}$;

(4) $\lim _{g \rightarrow f}\left\|\mathcal{L}_{f}^{*} \varphi-\mathcal{L}_{g}^{*} \varphi\right\|_{\infty}=0$ for all $\varphi \in C^{0}(X)$;

(5) The quantities $\tau_{f}(R)$ and $\beta_{f}(R)$, defined in (1) and (2), vary upper semicontinuously with $f$, for any set $R \subseteq X$.

In proposition 6.2 we give examples of topologies on $\mathcal{H}(X)$ which turn it into a topological semigroup of Markov systems. Then our main results are:

Theorem A (Spectral Stability Characterization)

Given a topological semigroup $\mathcal{H}_{1}$ of Markov systems, $f \in \mathcal{H}_{1}$ is spectrally stable if and only if $\varphi_{f}$ is combinatorially stable.

Theorem B (Genericity of Spectral Stability)

Given a topological semigroup $\mathcal{H}_{1}$ of Markov systems, the set of spectrally stable systems in $\mathcal{H}_{1}$ is open and dense in $\mathcal{H}_{1}$.

\section{Abstract Spectral Bounds}

Given $f \in \mathcal{H}(X)$ and an open $\varphi_{f}$-invariant set $R \subseteq X$, let

$$
\begin{aligned}
\tau_{f}(R) & =\frac{1}{2} \sup _{x, z \in R} \int_{R}|f(x, y)-f(z, y)| d m(y) \\
& =1-\min _{x, z \in R} \int_{R} f(x, y) \wedge f(z, y) d m(y)
\end{aligned}
$$

and

$$
\tau_{f}^{*}(R)=\inf _{n \geq 1}\left[\tau_{f^{n}}(R)\right]^{1 / n}
$$


The quantity $-\ln \left(\tau_{f}^{*}(R)\right)$ is a kind of mixing rate for the action of $\mathcal{L}_{f}$ on $\mathcal{M}_{\text {prob }}(R)$, which measures how fast the $\mathcal{L}_{f}$-iterates of any probability distribution on $R$ will converge to the unique $\mathcal{L}_{f}$-invariant measure supported in $R$. Next, we make some trivial remarks on this concept:

1. $\tau_{f^{n}}(R)=0 \Leftrightarrow$ the transition probabilities $f_{x}^{n}(\cdot)=f^{n}(x, \cdot)$ do not depend on $x$, for $x$ over $R$.

2. If for some pair of points $x, y \in R$, the transition probabilities $f_{x}^{n}$ and $f_{y}^{n}$ have disjoint supports, then $\tau_{f^{n}}(R)=1$.

3. If $\tau_{f}^{*}(R)<1$ then the restriction Markov system $f_{R}$ on $R$ is irreducible and acyclic.

Under the same invariance assumption on $R \subseteq X, \varphi_{f}(R) \subseteq R$, we define

$$
\begin{aligned}
\beta_{f}(R) & =1-\min _{x \in X} \int_{R} f(x, y) d m(y) \\
& =\sup _{x \in X} \int_{R^{c}} f(x, y) d m(y)
\end{aligned}
$$

and

$$
\beta_{f}^{*}(R)=\inf _{n \geq 1}\left[\beta_{f^{n}}(R)\right]^{1 / n} .
$$

The quantity $-\ln \left(\beta_{f}^{*}(R)\right)$ is a kind of escape rate, which measures how fast the restriction to $R^{c}$ of the $\mathcal{L}_{f}$-iterates of any probability distribution on $X$ will tend to zero. We also make some obvious remarks on this concept:

1. $\beta_{f^{n}}(R)=0 \Leftrightarrow \varphi_{f^{n}}(X)=\left(\varphi_{f}\right)^{n}(X) \subseteq R$.

2. If for some point $x \in X$, the transition probability $f_{x}^{n}$ has support disjoint from $R$, then $\beta_{f^{n}}(R)=1$.

3. If $\beta_{f}^{*}(R)<1$ then for every $x \in X$ the probability density $\left(f_{x}^{n}\right)_{R^{c}}$ converges to zero in $L_{R^{c}}^{1}$, as $n \rightarrow+\infty$. 
Given a subset $R \subseteq X$, we denote by $L_{R}^{1}$ the subspace of all functions $h \in L^{1}(X, m)$ which vanish outside $R$. We denote by $L_{R, 0}^{1}$ the subspace of functions $h \in L_{R}^{1}$ with zero average, i.e., $\int_{X} h(x) d m(x)=0$. Of course the subspaces $L_{R}^{1}$ and $L_{R, 0}^{1}$ are $\mathcal{L}_{f}$-invariant if and only if $R$ is $f$-invariant. The action of $\mathcal{L}_{f}$ on the invariant subspaces $L_{R}^{1}$ and $L_{R, 0}^{1}$ will be denoted by $\mathcal{L}_{f_{R}}$ and $\mathcal{L}_{f_{R, 0}}$, respectively. Assume, as before, that $R$ is $\varphi_{f}$-invariant. Then $L_{R}^{1}$ is $\mathcal{L}_{f}$-invariant. We can decompose $L^{1}(X, m)$ as $L^{1}(X, m)=L_{R}^{1} \oplus L_{R^{c}}^{1}$, where $R^{c}=X-R$. Given $h \in L^{1}(X, m)$, denote by $h_{R}=h \cdot \chi_{R}$ the function obtained multiplying $h$ by $R$ 's characteristic function $\chi_{R}$. Thus each function $q \in L^{1}(X, m)$ can be identified with the pair $\left[\begin{array}{c}q_{R} \\ q_{R^{c}}\end{array}\right]$. Using this notation, if $q^{\prime}=\left(\mathcal{L}_{f}\right)^{n} q$, for some $q \in L^{1}(X, m)$, then

$$
\left[\begin{array}{c}
q_{R}^{\prime} \\
q_{R^{c}}^{\prime}
\end{array}\right]=\left[\begin{array}{cc}
\left(\mathcal{L}_{f_{R}}\right)^{n} & \star \\
O & \left(\mathcal{L}_{f_{R^{c}}}\right)^{n}
\end{array}\right]\left[\begin{array}{c}
q_{R} \\
q_{R^{c}}
\end{array}\right],
$$

where $O$ denotes the null operator from $L_{R}^{1}$ into $L_{R^{c}}^{1}, \mathcal{L}_{f_{R}}$ describes the action of $\mathcal{L}_{f}$ on the invariant subspace $L_{R}^{1}$, and $\mathcal{L}_{f_{R c}}$ the action of $\mathcal{L}_{f}$ on the non-invariant subspace $L_{R^{c}}^{1}$ composed with the projection onto $L_{R^{c}}^{1}$. Notice that $\mathcal{L}_{f_{R^{c}}}$ is a PerronFrobenius type of operator associated with the sub-stochastic transition function $f_{R^{c}}$. Therefore, we can decompose the spectrum of $\mathcal{L}_{f}$ as

$$
\sigma\left(\mathcal{L}_{f}\right)=\sigma\left(\mathcal{L}_{f_{R}}\right) \cup \sigma\left(\mathcal{L}_{f_{R^{c}}}\right) .
$$

Because $R$ is $\varphi_{f}$-invariant, $\left(\mathcal{L}_{f_{R}}\right)^{n}=\mathcal{L}_{\left(f^{n}\right)_{R}} \quad$ and $\quad\left(\mathcal{L}_{f_{R^{c}}}\right)^{n}=\mathcal{L}_{\left(f^{n}\right)_{R^{c}}}$, this second equality because $\mathcal{L}_{f_{R^{c}}}(q)=\mathcal{L}_{f_{R^{c}}}\left(q_{R^{c}}\right)$.

Proposition 5.1. Given $f \in \mathcal{H}(X)$ and an open $\varphi_{f}$-invariant set $R \subseteq X$,

$$
\tau_{f}(R)=\sup _{q \in L_{R, 0}^{1}} \frac{\left\|\mathcal{L}_{f_{R}} q\right\|_{1}}{\|q\|_{1}}=\left\|\mathcal{L}_{f_{R, 0}}\right\|_{1} .
$$

In particular $\tau_{f}^{*}(R)=\lim _{n \rightarrow \infty}\left\|\left(\mathcal{L}_{f_{R, 0}}\right)^{n}\right\|_{1}{ }^{1 / n}=r\left(\mathcal{L}_{f_{R, 0}}\right)$ is the spectral radius of the operator $\mathcal{L}_{f_{R, 0}}$.

Proof. We denote by $f_{x}$ the function $y \mapsto f(x, y)$. By definition $\left\|f_{x}-f_{z}\right\|_{1} \leq 2 \tau_{f}(R)$, for every $x, z \in X$. Thus, given $q_{1}, q_{2} \in L_{R}^{1}$ such that $q_{1}, q_{2} \geq 0$ and $\int_{R} q_{1}(y) d m(y)=$ $\int_{R} q_{2}(y) d m(y)=1$, averaging $f_{x}$ with $q_{1}(x)$, and $f_{z}$ with $q_{2}(z)$, we obtain the inequality 
$\left\|\mathcal{L}_{f}\left(q_{1}\right)-\mathcal{L}_{f}\left(q_{2}\right)\right\|_{1} \leq 2 \tau_{f}(R)$. Now, given $q \in L_{R, 0}^{1}$, define $q^{+}:=\max \{q, 0\}, q^{-}:=$ $\max \{-q, 0\}$, and $\alpha:=\int_{R} q^{+}(y) d m(y)=\int_{R} q^{-}(y) d m(y)>0$. Notice that both $q_{1}=\alpha^{-1} q^{+}$and $q_{2}=\alpha^{-1} q^{-}$are probability densities. Therefore

$$
\left\|\mathcal{L}_{f}(q)\right\|_{1}=\alpha\left\|\mathcal{L}_{f}\left(q_{1}-q_{2}\right)\right\|_{1} \leq 2 \alpha \tau_{f}(R)=\tau_{f}(R)\|q\|_{1},
$$

which proves that $\left\|\mathcal{L}_{f_{R, 0}}\right\|_{1} \leq \tau_{f}(R)$. Conversely,

$$
\left\|f_{x}-f_{z}\right\|_{1}=\left\|\mathcal{L}_{f}\left(\delta_{x}\right)-\mathcal{L}_{f}\left(\delta_{z}\right)\right\|_{1} \leq\left\|\mathcal{L}_{f_{R, 0}}\right\|_{1}\left\|\delta_{x}-\delta_{z}\right\|=2\left\|\mathcal{L}_{f_{R, 0}}\right\|_{1},
$$

where $\|\cdot\| \|$ stands for the total variation norm on finite measures. This inequality can be proved using approximate identities for the Dirac measures $\delta_{x}$ and $\delta_{z}$. This proves that $\tau_{f}(R) \leq\left\|\mathcal{L}_{f_{R, 0}}\right\|_{1}$ and concludes the proof.

Proposition 5.2. Given $f \in \mathcal{H}(X)$ and an open $\varphi_{f}$-invariant set $R \subseteq X$,

$$
\beta_{f}(R)=\sup _{q \in L^{1}(X, m)} \frac{\left\|\mathcal{L}_{f_{R^{c}}} q\right\|_{1}}{\|q\|_{1}}=\left\|\mathcal{L}_{f_{R^{c}}}\right\|_{1} .
$$

In particular $\beta_{f}^{*}(R)=\lim _{n \rightarrow \infty}\left\|\left(\mathcal{L}_{f_{R^{c}}}\right)^{n}\right\|_{1}^{1 / n}=r\left(\mathcal{L}_{f_{R^{c}}}\right)$ is the spectral radius of the operator $\mathcal{L}_{f_{R^{c}}}$.

Corollary 5.3. Given $f \in \mathcal{H}(X)$ and an open $\varphi_{f}$-invariant set $R \subseteq X$,

$$
r_{\text {int }}\left(\mathcal{L}_{f}\right) \leq \max \left\{\tau_{f}^{*}(R), \beta_{f}^{*}(R)\right\} .
$$

The next five propositions and corollaries relate the spectral behavior of $\mathcal{L}_{f}$ with the asymptotics of $f^{n}(x, y)$ as $n \rightarrow \infty$. Although we state them in our mixed measuretopological context, they could be restated in more abstract terms, as pure measuretheoretical statements. See [D]. Given $f \in \mathcal{H}(X)$ and a set $R \subseteq X$ define

$$
\left.\sup _{R} f\right)(y)=\sup _{x \in R} f(x, y), \quad\left(\inf _{R} f\right)(y)=\inf _{x \in R} f(x, y),
$$

and the oscillation of $f$ on $R$ to be

$$
\omega_{f, R}(y)=\sup _{x, z \in R}|f(x, y)-f(z, y)|=\left(\sup _{R} f\right)(y)-\left(\inf _{R} f\right)(y) .
$$


Proposition 5.4. Given $f \in \mathcal{H}(X)$ and an open $\varphi_{f}$-invariant set $R \subseteq X$,

$$
\omega_{f^{n+m}, R}(y) \leq \tau_{f^{m}}(R) \omega_{f^{n}, R}(y) \quad \forall n, m \geq 1, y \in R .
$$

If $\tau_{f}^{*}(R) \leq r<1$ then there is some constant $C>0$ and a lower semicontinuous function $q \in L_{R}^{1}$ such that $q=\mathcal{L}_{f} q, \omega_{f^{n}, R}(y) \leq C r^{n} \quad$ and $\quad\left|f^{n}(x, y)-q(y)\right| \leq C r^{n}$ for all $n \geq 1$ and $(x, y) \in R \times R$. Furthermore, if $f$ is continuous then so is $q$.

Proof. Assume $\tau_{f}^{*}(R) \leq r<1$. By proposition 5.1 the operator $\mathcal{L}_{f}$ acts contractively on the affine space of probability densities in $L_{R}^{1}$. Thus, there is a unique probability density $q \in L_{R_{i}}^{1}$ such that $f^{n}(x, \cdot)=\mathcal{L}_{f}^{n-1}(f(x, \cdot))$ converges in $L^{1}$ to $q$ as $n \rightarrow \infty$. It follows that $f^{n}(x, \cdot)$ converges almost everywhere to $q$. By taking the limit we get $q=$ $\mathcal{L}_{f}(q)$, which implies that $q$ is lower semicontinuous, and continuous when so is $f$. The oscillation inequality can be proved adapting an argument from [D], see p. 197, with the strengthened Doeblin condition replaced by $\tau_{f}^{*}(R) \leq r$. For $x, z \in R$ define $B_{f, R}^{+}=$ $\{y \in R: f(x, y)-f(z, y)>0\}$ and $B_{f, R}^{-}=\{y \in R: f(x, y)-f(z, y)<0\}$. Since $\int_{R} f(x, y) d y=\int_{R} f(z, y) d y=1$, we have $\int_{B_{f, R}^{+}} f(x, y)-f(z, y) d y=-\int_{B_{f, R}^{-}} f(x, y)-$ $f(z, y) d y$. Thus

$$
\begin{gathered}
f^{n+m}(x, y)-f^{n+m}(z, y)=\int_{R}\left(f^{m}(x, v)-f^{m}(z, v)\right) f^{n}(v, y) d v \\
\leq \sup _{R} f^{n}(y) \int_{B_{f^{m}, R}^{+}} f^{m}(x, v)-f^{m}(z, v) d v \\
\quad+\inf _{R} f^{n}(y) \int_{B_{f^{m}, R}^{-}} f^{m}(x, v)-f^{m}(z, v) d v \\
=\omega_{f^{n}, R}(y) \int_{B_{f^{m}, R}^{+}} f^{m}(x, v)-f^{m}(z, v) d v
\end{gathered}
$$

Finally, since

$$
\int_{B_{f^{m}, R}^{+}} f^{m}(x, v)-f^{m}(z, v) d v=\frac{1}{2} \int_{R}\left|f^{m}(x, v)-f^{m}(z, v)\right| d v \leq \tau_{f^{m}}(R),
$$

we get that $\omega_{f^{n+m}, R}(y) \leq \tau_{f^{m}}(R) \omega_{f^{n}, R}(y)$. This inequality shows that $\omega_{f^{n}, R}(y)$ decreases to zero uniformly at a geometric rate dominated by $r^{n}$. It follows that all $f^{n}(x, \cdot)$ converge to $q$ at the same geometric rate. 
Proposition 5.5. Given $f \in \mathcal{H}(X)$ and an open $\varphi_{f}$-invariant set $R \subseteq X$,

$$
\sup _{X} f^{n+m}(y) \leq \beta_{f^{m}}(R) \sup _{X} f^{n}(y) \quad \forall n, m \geq 1, y \in R^{c} .
$$

If $\beta_{f}^{*}(R) \leq r<1$ then there is some constant $C>0$ such that $0 \leq f^{n}(x, y) \leq C r^{n}$ for all $n \geq 1$ and $(x, y) \in X \times R^{c}$.

Proof. Take $\beta_{f}^{*}(R) \leq r<1$. Given $y \in R^{c}$, because $R$ is $\varphi_{f}$-invariant we have

$$
\begin{aligned}
\sup _{X} f^{n+m}(y) & =\sup _{x \in X} \int_{X} f^{m}(x, z) f^{n}(z, y) d z \\
& =\sup _{x \in X} \int_{R^{c}} f^{m}(x, z) f^{n}(z, y) d z \\
& \leq \sup _{X} f^{n}(y) \sup _{x \in X} \int_{R^{c}} f^{m}(x, z) d z \\
& =\sup _{X} f^{n}(y) \beta_{f^{m}}(R) .
\end{aligned}
$$

This proves that $f^{n}(x, y)$ decreases to zero at a geometric rate dominated by $r^{n}$.

We shall say that an open $\varphi_{f}$-invariant set $R \subseteq X$ is an acyclic spectral attractor for $f \in \mathcal{H}(X)$ if and only if $R$ is connected and $\tau_{f}^{*}(R)<1$. When the set $R$ splits as a disjoint union of $d$ connected sets,

$$
R=R_{0} \cup \varphi_{f}\left(R_{0}\right) \cup \cdots \cup \varphi_{f}^{d}\left(R_{0}\right),
$$

such that $R_{0}$ is an acyclic spectral attractor for $f^{d}$ we say that $R$ is a periodic spectral attractor of period $d$. We shall say that an open $\varphi_{f}$-invariant set $R \subseteq X$ is spectrally attractive for $f \in \mathcal{H}(X)$ if and only if $\beta_{f}^{*}(R)<1$.

Corollary 5.6. Let $R \subseteq X$ be a periodic spectral attractor of period d for $f \in \mathcal{H}(X)$ with connected components $R_{0}, \cdots, R_{d-1}$. For each component $R_{i},(0 \leq i \leq d-1)$ there exists a unique $\mathcal{L}_{f^{d}}$-invariant probability density $q_{i}$ supported in $R_{i}$ such that for every $(x, y) \in R_{i} \times R_{i}$,

$$
q_{i}(y)=\lim _{n \rightarrow \infty} f^{n d}(x, y) .
$$


Furthermore, given $\tau_{f^{d}}^{*}\left(R_{i}\right)<r<1$ there is $C_{r}(f)>0$ such that for all $(x, y) \in$ $R_{i} \times R_{i}$ and $n \geq 1$

$$
\left|f^{n d}(x, y)-q_{i}(y)\right| \leq C_{r} r^{n} .
$$

Moreover $q_{i}$ is lower semi-continuous and is continuous when $f$ is continuous.

Proof. Apply proposition 5.4 to each $f^{d}$-invariant set $R_{i}$.

Corollary 5.7. Let $R \subseteq X$ be spectrally attractive for $f \in \mathcal{H}(X)$. For every $(x, y) \in$ $X \times R^{c}$

$$
\lim _{n \rightarrow \infty} f^{n}(x, y)=0 .
$$

Furthermore, given $\beta_{f}^{*}(R)<r<1$ there is $C_{r}(f)>0$ such that for every $(x, y) \in X \times R^{c}$

$$
0 \leq f^{n}(x, y) \leq C_{r} r^{n}
$$

Proof. Follows from proposition 5.5.

Corollary 5.8. If $\tau_{f}^{*}(R)<1$ and $\beta_{f}^{*}(R)<1$ then $f$ has a unique invariant measure supported in $R, \mu=\mathcal{L}_{f}(\mu)$, which is an attractive fixed point for the the affine action of operator $\mathcal{L}_{f}$ on $\mathcal{M}_{\text {prob }}(X)$.

Finally, we extract some spectral information on $\mathcal{L}_{f}$ from the combinatorics of $\varphi_{f}$. Notice that, unlike the previous, these propositions can not be restated in pure measure-theoretical terms.

Proposition 5.9. Given $f \in \mathcal{H}(X)$, each $\Omega$-final class of period $d$ is a periodic spectral attractor of period $d$ for $f$.

Proposition 5.10. Given $f \in \mathcal{H}(X), \Omega_{\mathrm{final}}\left(\varphi_{f}\right)$ is spectrally attractive for $f$. 
Corollary 5.11. Given $f \in \mathcal{H}(X)$, let $\Sigma_{\text {final }}^{\Omega}\left(\varphi_{f}\right)=\left\{R_{1}, R_{2}, \cdots, R_{s}\right\}$, $R$ be a $\varphi_{f^{-}}$ invariant neighborhood of $\Omega_{\mathrm{final}}\left(\varphi_{f}\right)$, and $\kappa_{f}$ be the maximum between $\beta_{f}^{*}(R)$ and $\tau_{f}^{*}\left(R_{i}\right)$, for $i=1, \cdots, s$. Then

$$
r_{\text {int }}\left(\mathcal{L}_{f}\right) \leq \kappa_{f} .
$$

Corollary 5.12. Given $f \in \mathcal{H}(X)$, let $\Sigma_{\text {final }}^{\Omega}\left(\varphi_{f}\right)=\left\{R_{1}, R_{2}, \cdots, R_{s}\right\}$, where each component $R_{i}$ is $\varphi_{f}^{d_{i}}$-invariant for some power $d_{i} \geq 1$. Then there is a $f^{d_{i}}$-invariant measure supported on $R_{i}, \mu_{i}=\mathcal{L}_{f^{d_{i}}} \mu_{i}$, for each $i=1, \cdots, s$, such that:

1. The sum $E_{1}(f)$ of all generalized eigenspaces associated with eigenvalues in the unit circle is the $s$-dimensional space spanned by the measures $\mu_{1}, \cdots, \mu_{s}$.

2. The action of $\mathcal{L}_{f}$ on the invariant subspace $E_{1}(f)$ w.r.t. the basis $\left\{\mu_{1}, \cdots, \mu_{s}\right\}$ is represented by the permutation matrix associated with the permutation $\pi_{\varphi_{f}}$.

3. The eigenvalues of $\mathcal{L}_{f}$ in the unit circle are, with multiplicity, the $d$-unity roots $\mathbb{U}^{d}=\left\{\lambda \in \mathbb{C}: \lambda^{d}=1\right\}$, counted for every cycle of length $d$ in permutation $\pi_{\varphi_{f}}$.

4. The operator induced by $\mathcal{L}_{f}$ on the quotient $\mathcal{M}_{\mathrm{prob}}(X) / E_{1}(f)$ is contractive, i.e., it has norm less than one.

To prove Proposition 5.9 and Proposition 5.10 above we use the concept of final kernel defined in a previous article. In [DT] we called thickness of $\varphi_{f}$ to the smallest volume ( $m$-measure) of all components in $\Sigma_{\text {final }}^{\Omega}\left(\varphi_{f}\right)$. We said that an open set $K \subseteq$ $\Omega_{\text {final }}\left(\varphi_{f}\right)$ is a final kernel of $\varphi_{f}$ if and only if there is a one-to-one correspondence $R \mapsto K_{R}$, between components $R \in \Sigma_{\text {final }}^{\Omega}(\varphi)$ and connected components $K_{R}$ of $K$, such that $\overline{K_{R}} \subseteq R$ for every $R \in \Sigma_{\text {final }}^{\Omega}\left(\varphi_{f}\right)$. We said that $K$ is a final kernel with finite order $N$ if and only if $K$ is a final kernel of $\varphi_{f}$, and furthermore

(1) For each component $R \in \Sigma_{\text {final }}^{\Omega}\left(\varphi_{f}\right)$ of period $d$, the only connected component $K_{R}$ of $K$ contained in $R$ satisfies $\overline{R \times K_{R}} \subseteq \operatorname{graph}\left(\varphi_{f}^{N d}\right)$.

(2) For each $x \in X, \varphi_{f}^{N}(x)$ contains at least the closure of one of $K$ 's connected components.

We did call thickness of $K$ to the smallest volume of all connected components of $K$. We proved that every final kernel $K$ of $\varphi_{f}$ is a final kernel with some finite order $N \in \mathbb{N}$. In particular, $\varphi_{f}$ admits finite-order final kernels, whose thickness is arbitrarily close to the thickness of $\varphi_{f}$. See Lemma 5.20 of [DT]. 
Proof. of proposition 5.9 Let $R_{1}, \cdots, R_{s}$ be the $\Omega$-final components of $\varphi_{f}$. Take some final kernel $K \subseteq \Omega_{\text {final }}\left(\varphi_{f}\right)$ and choose $N \in \mathbb{N}$ such that $K$ is a finite kernel of order $N$. Let $K_{1}, \cdots, K_{s}$ be the connected components of $K$ corresponding to $R_{1}, \cdots, R_{s}$. Item 1 . in the definition of finite kernel of order $N$ implies that for each component $R_{i}$ of period $d_{i}$ and for all $(x, y) \in \overline{R_{i} \times K_{i}}$, one has $f^{N d_{i}}(x, y)>$ 0 . Moreover, because $f$ is lower semi-continuous, there is $c_{i}>0$ such that for all $(x, y) \in \overline{R_{i} \times K_{i}}, f^{N d_{i}}(x, y) \geq c_{i}$. Therefore, $\tau_{f^{N d_{i}}}\left(R_{i}\right) \leq 1-c_{i}<1$ which implies that $\tau_{f^{d_{i}}}^{*}\left(R_{i}\right)<1$.

Proof. of proposition 5.10 Take some final kernel $K \subseteq \Omega_{\text {final }}\left(\varphi_{f}\right)$ and choose $N \in \mathbb{N}$ such that $K$ is a finite kernel of order $N$. Item 2. in the definition of finite kernel of order $N$ implies that for each $x \in X, \int_{K} f^{N}(x, y) d m(y)>0$. Thus, since $K \subseteq \Omega_{\text {final }}\left(\varphi_{f}\right)$, one has $\int_{\Omega_{\text {final }}\left(\varphi_{f}\right)} f^{N}(x, y) d m(y)>0$ for each $x \in X$. Moreover, because $f$ is lower semi-continuous, there is $\alpha_{0}>0$ such that for all $x \in X$, $\int_{\Omega_{\mathrm{final}}\left(\varphi_{f}\right)} f^{N}(x, y) d m(y) \geq \alpha_{0}$. Therefore, $\beta_{f^{N}}\left(\Omega_{\mathrm{final}}\left(\varphi_{f}\right)\right) \leq 1-\alpha_{0}<1$ and, consequently, $\beta_{f}^{*}\left(\Omega_{\text {final }}\left(\varphi_{f}\right)\right)<1$.

\section{Topological Semigroups of Markov Systems}

We now topologize the semigroup $\mathcal{H}(X)$ turning it into a topological semigroup of Markov systems. Consider

$$
d_{\infty}(f, g)=\max _{(x, y) \in X \times X}|f(x, y)-g(x, y)|
$$

and

$$
d_{1}(f, g)=\max _{x \in X} \int_{X}|f(x, y)-g(x, y)| d m(y)
$$

Proposition 6.1. Given $f, g \in \mathcal{H}(X)$ and $N \in \mathbb{N}$,

1. $\left\|f^{N}\right\|_{\infty} \leq\|f\|_{\infty}$

2. $\left\|f^{N}\right\|_{1} \leq\|f\|_{1}$;

3. $d_{\infty}\left(f^{N}, g^{N}\right) \leq d_{\infty}(f, g)\left(1+(N-1)\|f\|_{\infty}\right)$; 


$$
\text { 4. } d_{1}\left(f^{N}, g^{N}\right) \leq d_{1}(f, g)\left(1+(N-1)\|f\|_{\infty}\right) \text {. }
$$

Proof. We notice that

$$
f * g(x, \cdot)=\mathcal{L}_{f}(g(x, \cdot)) \quad \text { and } \quad f * g(\cdot, y)=\mathcal{L}_{f}^{*}(g(\cdot, y))
$$

Using that both operators $\mathcal{L}_{f}$ and $\mathcal{L}_{f}^{*}$ have norm equal to one, it follows that both sequences $\left\|f^{N}\right\|_{1}$ and $\left\|f^{N}\right\|_{\infty}$ decrease, which proves 1 . and 2.. For the metrics $d=d_{1}$ and $d=d_{\infty}$ we use the triangle inequality

$$
d\left(f^{N}, g^{N}\right) \leq d\left(f * f^{N-1}, g * f^{N-1}\right)+d\left(g * f^{N-1}, g * g^{N-1}\right)
$$

and the same kind of argument as above, to derive inequalities 3. and 4 ..

Given $f, g \in \mathcal{H}(X)$ we say that $f$ is dominated by $g$ when $\varphi_{f} \prec \varphi_{g}$.

Proposition 6.2. Consider any sub-semigroup $\mathcal{O}_{1} \subseteq \mathcal{O}(X)$ with a topology defined by some metric $\rho$ which makes it a topological semigroup of open maps. Define

$$
\rho_{\infty}(f, g)=\max \left\{d_{\infty}(f, g), \rho\left(\varphi_{f}, \varphi_{g}\right)\right\}
$$

and

$$
\rho_{1}(f, g)=\max \left\{d_{1}(f, g), \rho\left(\varphi_{f}, \varphi_{g}\right)\right\} .
$$

Then $\mathcal{H}_{1}=\left\{f \in \mathcal{H}(X): \varphi_{f} \in \mathcal{O}_{1}\right\}$ with the topology associated with any of the metrics $\rho_{\infty}$ and $\rho_{1}$, is a topological semigroup of Markov systems over $\mathcal{O}_{1}$.

Proof. Consider $\mathcal{H}_{1}$ with any of the topologies induced by the metrics above. We need to prove that $\mathcal{H}_{1}$ satisfies items (1) to (5) of definition 4.2. Items (1) and (2) are clear. Let us now prove item(3). Take $f \in \mathcal{H}_{1}$. By Definition 2.1, for each $\epsilon>0$ we can take $\varphi_{\epsilon}^{*} \in \mathcal{O}_{1}$ such that $\varphi_{f} \prec \varphi_{\epsilon}^{*}$ and $\lim _{\epsilon \rightarrow 0^{+}} \rho\left(\varphi_{f}, \varphi_{\epsilon}^{*}\right)=0$. Let $\left\{h_{\epsilon}\right\}_{\epsilon>0}, h_{\epsilon}: X \times X \rightarrow[0,1]$, be a family of continuous maps such that:

(i) $\operatorname{graph}\left(\varphi_{h_{\epsilon}}\right)=\operatorname{graph}\left(\varphi_{\epsilon}^{*}\right)$, and

(ii) $h_{\epsilon}(x, y)=1$ for every $(x, y) \in \operatorname{graph}\left(\varphi_{f}\right)$. 
For each map $h_{\epsilon}$ and $x \in X$, let

$$
\tilde{h}_{\epsilon}(x)=\int_{X} h_{\epsilon}(x, y) d m(y),
$$

and define, for $x, y \in X$,

$$
f_{\epsilon}(x, y)=\frac{h_{\epsilon}(x, y)}{\tilde{h}_{\epsilon}(x)} .
$$

For every $x \in X, \tilde{h}_{\epsilon}(x) \geq m\left(\varphi_{f}(x)\right) \geq c_{0}>0$, where $c_{0}$ denotes the volume ( $m$ measure) of a ball of radius $\xi_{0}>0$, for some $\xi_{0}$ depending on $\varphi_{f}$. (See Lemma 2.7 in [DT] where we prove that given $\varphi \in \mathcal{O}(X)$ there is a map $F: X \rightarrow X$ and $\xi_{0}>0$ such that the $\xi_{0}$-radius ball of graph $(F)$ is contained in graph $\left.(\varphi)\right)$. Therefore, $f_{\epsilon}$ is bounded and continuous. We define the family $\left\{g_{\epsilon}\right\}_{\epsilon>0}$ by $g_{\epsilon}=(1-\epsilon) f+\epsilon f_{\epsilon}$. It is easy to see that $g_{\epsilon} \in \mathcal{H}(X)$. Clearly, $\lim _{\epsilon \rightarrow 0} d_{\infty}\left(f, g_{\epsilon}\right)=0$, which implies that $\lim _{\epsilon \rightarrow 0} d_{1}\left(f, g_{\epsilon}\right)=0$. Moreover, $\varphi_{g_{\epsilon}}$ is an open map whose graph coincides with the graph of $\varphi_{\epsilon}^{*}$. Therefore, $\lim _{\epsilon \rightarrow 0} \rho\left(\varphi_{f}, \varphi_{g_{\epsilon}}\right)=0$ and $\lim _{\epsilon \rightarrow 0} \rho_{\infty}\left(f, g_{\epsilon}\right)=\lim _{\epsilon \rightarrow 0} \rho_{1}\left(f, g_{\epsilon}\right)=0$.

To prove item (4) of definition 4.2 notice that given $f, g \in \mathcal{H}_{1}$, for all $\varphi \in C^{0}(X)$ :

$$
\begin{aligned}
\left\|\mathcal{L}_{f}^{*} \varphi-\mathcal{L}_{g}^{*} \varphi\right\|_{\infty} & =\max _{x \in X}\left|\mathcal{L}_{f}^{*} \varphi(x)-\mathcal{L}_{g}^{*} \varphi(y)\right| \\
& =\max _{x \in X}\left|\int_{X} f(x, y) \varphi(y) d m(y)-\int_{X} g(x, y) \varphi(y) d m(y)\right| \\
& \leq \max _{x \in X} \int_{X}|f(x, y)-g(x, y)||\varphi(y)| d m(y) \\
& \leq \max _{y \in X}|\varphi(y)| \max _{x \in X} \int_{X}|f(x, y)-g(x, y)| d m(y) \\
& =\max _{y \in X}|\varphi(y)| d_{1}(f, g) \\
& \leq \max _{y \in X}|\varphi(y)| d_{\infty}(f, g) .
\end{aligned}
$$

Finally, we prove item (5) of definition 4.2. Given $f, g \in \mathcal{H}_{1}$ and open $\varphi_{f}$-invariant 
set $R \subseteq X$ we have that:

$$
\begin{aligned}
\left|\tau_{f}(R)-\tau_{g}(R)\right| & =\left|\min _{x, z \in R} \int_{R} f(x, y) \wedge f(z, y) d m(y)-\min _{x, z \in R} \int_{R} f(x, y) \wedge g(z, y) d m(y)\right| \\
& \leq\left|\min _{x \in R} \int_{R} f(x, y) d m(y)-\min _{x \in R} \int_{R} g(x, y) d m(y)\right| \\
& \leq \max _{x \in R} \int_{R}|f(x, y)-g(x, y)| d m(y) \\
& =d_{1}(f, g) \leq d_{\infty}(f, g)
\end{aligned}
$$

and

$$
\begin{aligned}
\left|\beta_{f}(R)-\beta_{g}(R)\right| & =\left|\min _{x \in X} \int_{R} f(x, y) d m(y)-\min _{x \in X} \int_{R} g(z, y) d m(y)\right| \\
& \leq \max _{x \in X} \int_{R}|f(x, y)-g(x, y)| d m(y) \\
& =d_{1}(f, g) \leq d_{\infty}(f, g) .
\end{aligned}
$$

\section{Spectral Stability}

In this final section we prove theorems A and B.

Proposition 7.1. Let $\mathcal{H}_{1}$ be any topological semigroup of Markov systems. Given $f \in \mathcal{H}_{1}$ with $\varphi_{f}$ combinatorially stable, let $R_{1}, \cdots, R_{s}$ be the $\Omega$-final components of $\varphi_{f}$. There is an open neighborhood $\mathcal{N}$ of $f$ in $\mathcal{H}_{1}$ and there are open neighborhoods $U_{1}, \cdots, U_{s}$ of $R_{1}, \cdots, R_{s}$, respectively, such that

(1) Each $U_{i},(1 \leq i \leq s)$, is an acyclic spectral attractor for $f^{d_{i}}$ for some $d_{i} \geq 1$.

(2) For all $g \in \mathcal{N}, \varphi_{g}^{d_{i}}\left(U_{i}\right) \subseteq U_{i} \quad(1 \leq i \leq s)$. 
Proof. Since $\varphi_{f}$ is combinatorially stable there is a neighborhood $\mathcal{U}$ of $\varphi_{f}$ in $\mathcal{O}_{1}$ such that every $\phi \in \mathcal{U}$ is combinatorially equivalent to $\varphi_{f}$. By Definition 4.2(2) the map $f \mapsto \varphi_{f}$ is continuous and, therefore, there is a neighborhood $\mathcal{N}$ of $f$ in $\mathcal{H}_{1}$ such that for every $h \in \mathcal{N}, \varphi_{h} \in \mathcal{U}$. Furthermore, Definition 4.2(3) ensures that there is $h \in \mathcal{N}$ such that $\varphi_{f} \prec \varphi_{h}$. Let $U_{1}, \cdots, U_{s}$ be the $\Omega$-final components of $\varphi_{h}$, i.e. $\Sigma_{\text {final }}^{\Omega}\left(\varphi_{h}\right)=\left\{U_{1}, U_{2}, \cdots, U_{s}\right\}$. Because $\varphi_{f}$ is combinatorially equivalent to $\varphi_{h}$ and $\varphi_{f} \prec \varphi_{h}$ we can sort the $U_{i}$ so that $R_{i} \subseteq U_{i}$, for $i=1, \cdots, s$. Take some final kernel $K \subseteq \Omega_{\text {final }}\left(\varphi_{h}\right)$ and choose $N \in \mathbb{N}$ such that $K$ is a finite kernel of order $N$. Let $K_{1}, \cdots, K_{s}$ be the connected components of $K$ corresponding to $U_{1}, \cdots, U_{s}$. Item 1 . in the definition of finite kernel of order $N$ implies that that for all $(x, y) \in \overline{U_{i} \times K_{i}}$, one has $f^{N d_{i}}(x, y)>0$. Moreover, because $f$ is lower semi-continuous, there is $c_{i}>0$ such that for all $(x, y) \in \overline{U_{i} \times K_{i}}, f^{N d_{i}}(x, y) \geq c_{i}$. Therefore, $\tau_{f^{N d_{i}}}\left(U_{i}\right) \leq 1-c_{i}<1$ which implies that $\tau_{f_{i}}^{*}\left(U_{i}\right)<1$.

To prove item 2. choose some neighborhood $\mathcal{N}$ of $f$ in $\mathcal{H}_{1}$ such that for all $g \in \mathcal{N}$, graph $\left(\varphi_{g}\right) \subseteq$ graph $\left(\varphi_{h}\right)$. Such a neighborhood exists by Definition 2.1(1) and Definition 4.2(2). Therefore $\varphi_{g}^{d_{i}}\left(U_{i}\right) \subseteq \varphi_{h}^{d_{i}}\left(U_{i}\right) \subseteq U_{i}$.

Lemma 7.2. Given $\varphi, \psi \in \mathcal{O}(X)$, such that $\varphi \prec \psi$, if $\varphi$ is combinatorially equivalent to $\psi$ then $\varphi$ is combinatorially stable.

Proof. Since $\varphi \prec \psi$, it is a straightforward consequence of the definitions that we have:

$$
\left|\Lambda_{\text {final }}^{\Omega}(\varphi)\right| \geq\left|\Lambda_{\text {final }}^{R}(\varphi)\right| \geq\left|\Lambda_{\text {final }}^{\Omega}(\psi)\right|
$$

and

$$
\left|\Sigma_{\text {final }}^{\Omega}(\varphi)\right| \geq\left|\Sigma_{\text {final }}^{R}(\varphi)\right| \geq\left|\Sigma_{\text {final }}^{\Omega}(\psi)\right| .
$$

Because $\varphi$ is combinatorially equivalent to $\psi$ we have that $\left|\Lambda_{\text {final }}^{\Omega}(\varphi)\right|=\left|\Lambda_{\text {final }}^{\Omega}(\psi)\right|$ and $\left|\Sigma_{\text {final }}^{\Omega}(\varphi)\right|=\left|\Sigma_{\text {final }}^{\Omega}(\psi)\right|$. Therefore, $\left|\Lambda_{\text {final }}^{\Omega}(\varphi)\right|=\left|\Lambda_{\text {final }}^{R}(\varphi)\right|$ and $\left|\Sigma_{\text {final }}^{\Omega}(\varphi)\right|=$ $\left|\Sigma_{\text {final }}^{R}(\varphi)\right|$, that is, $\varphi$ satisfies the combinatorially stability condition, which implies that $\varphi$ is combinatorially stable.

Proof. of Theorem A (Spectral Stability Characterization) Given $f \in \mathcal{H}_{1}$ assume $\varphi_{f}$ is combinatorially stable. By Definition 4.2(2) there is a neighborhood $\mathcal{N}$ of $f$ in $\mathcal{H}_{1}$ such that for all $g \in \mathcal{N}, \varphi_{g}$ is combinatorially equivalent to $\varphi_{f}$. Consequently it follows from Corollary 5.12 that there is a map $h_{g}: E_{1}(f) \rightarrow E_{1}(g)$ that conjugates $\left.\mathcal{L}_{f}\right|_{E_{1}(f)}$ to $\left.\mathcal{L}_{g}\right|_{E_{1}(g)}$, which proves Definition 4.1(1). 
To prove item (2) of Definition 4.1 we just need to note that Definition $4.2(4)$ easily implies that the invariant measures of $\mathcal{L}_{f}$ vary continuously with $f$ w.r.t. the weak-* topology. Indeed, given $f \in \mathcal{H}$ with $\varphi_{f}$ combinatorially stable, let $\mathcal{N}$ and $U_{i}, 1 \leq i \leq s$, be as in Proposition 7.1. We have that every invariant measure of $\mathcal{L}_{g}$ with $g \in \mathcal{N}$ is supported in some $\overline{U_{i}}$. Take $\mu_{f} \in E_{1}(f)$ and $\mu_{g} \in E_{1}(g)$ with $\mu_{f}\left(U_{i}\right)=\mu_{g}\left(U_{i}\right)=1$. Choose $N \in \mathbb{N}$ such that $\left\|\mathcal{L}_{f_{U_{i}, 0}^{N}}\right\|_{1}<1$. For any $\varphi \in C^{0}(X)$,

$$
\begin{aligned}
\left|\left\langle\mu_{f}-\mu_{g}, \varphi\right\rangle\right| & =\left|\left\langle\mathcal{L}_{f^{N}} \mu_{f}-\mathcal{L}_{g^{N}} \mu_{g}, \varphi\right\rangle\right| \\
& \leq\left|\left\langle\mathcal{L}_{f^{N}} \mu_{f}, \varphi\right\rangle-\left\langle\mathcal{L}_{f^{N}} \mu_{g}, \varphi\right\rangle\right|+\left|\left\langle\mathcal{L}_{f^{N}} \mu_{g}, \varphi\right\rangle-\left\langle\mathcal{L}_{g^{N}} \mu_{g}, \varphi\right\rangle\right| \\
& \leq\left|\left\langle\mathcal{L}_{f^{N}}\left(\mu_{f}-\mu_{g}\right), \varphi\right\rangle\right|+\left|\left\langle\left(\mathcal{L}_{f^{N}}-\mathcal{L}_{g^{N}}\right) \mu_{g}, \varphi\right\rangle\right|
\end{aligned}
$$

Definition 4.2(4) implies that

$$
\lim _{g \rightarrow f}\left|\left\langle\left(\mathcal{L}_{f^{N}}-\mathcal{L}_{g^{N}}\right) \mu_{g}, \varphi\right\rangle\right|=0
$$

Therefore,

$$
\lim _{g \rightarrow f}\left|\left\langle\mu_{f}-\mu_{g}, \varphi\right\rangle\right|=0
$$

which proves item (2) of Definition 4.1.

Let $R_{1}, \cdots, R_{s}$ be the $\Omega$-final components of $\varphi_{f}$ in $\Sigma_{\text {final }}^{\Omega}\left(\varphi_{f}\right)$. By Proposition 7.1(1) for each $\Omega$-component $R_{i},(1 \leq i \leq s)$, there is an open neighborhood $U_{i}$ of $R_{i}$ such that $U_{i}$ is an acyclic spectral attractor for $f^{d_{i}}$ for some power $d_{i} \geq 1$. In particular, $\tau_{f^{d_{i}}}^{*}\left(U_{i}\right)<1$ which implies that $\tau_{f}^{*}\left(U_{i}\right)<1$. Set $U=\cup_{i=1}^{s} U_{i}$. It is clear that $\Omega_{\text {final }}\left(\varphi_{f}\right) \subseteq U$. Furthermore, because $\Omega_{\text {final }}\left(\varphi_{f}\right)$ is spectrally attractive, one has $\beta_{f}^{*}\left(\Omega_{\text {final }}(\varphi)\right)<1$. These two facts together imply that $\beta_{f}^{*}(U)<1$. By Corollary 5.11 we have that $r_{\text {int }}\left(\mathcal{L}_{f}\right)<1$.

By Proposition 7.1(2) we can make $\mathcal{N}$ smaller so that for every $g \in \mathcal{N}$, one has $\varphi_{g^{d_{i}}}\left(U_{i}\right) \subseteq U_{i},(1 \leq i \leq s)$. Thus, it follows immediately from Corollary 5.3 that

$$
r_{\text {int }}\left(\mathcal{L}_{g}\right) \leq \max \left\{\left\{\tau_{g}^{*}\left(U_{i}\right), 1 \leq i \leq s\right\} \cup\left\{\beta_{g}^{*}(U)\right\}\right\} .
$$

By Definition 4.2(5) $\tau_{f}^{*}\left(U_{i}\right)$ and $\beta_{f}^{*}(U)$ vary upper semicontinuously with $f$. Therefore we can make $\mathcal{N}$ even smaller so that there is $k<1$ such that for every $g \in \mathcal{N}$, one has $r_{\text {int }}\left(\mathcal{L}_{g}\right) \leq k$. This proves Definition $4.1(3)$ that there is a spectral gap of size $k$ isolating $\sigma_{1}\left(\mathcal{L}_{g}\right)$ and $\sigma_{0}\left(\mathcal{L}_{g}\right)$. Therefore, $f$ is spectrally stable. 
Assume now that $f$ is $\mathcal{H}_{1}$-spectrally stable and let $\mathcal{N}$ be a neighborhood of $f$ in $\mathcal{H}_{1}$ where all the systems are spectrally equivalent. In particular, by Definition 4.1(1), all maps $\varphi_{g}$ with $g \in \mathcal{N}$ are combinatorially equivalent. By Definition 4.2(3) there is $g \in \mathcal{N}$ such that $\varphi_{f} \prec \varphi_{g}$. Therefore, by Lemma 7.2, $\varphi_{f}$ is combinatorially stable.

Proof. of Theorem B (Genericity of Spectral Stability) By definition, spectral stability is an open property. Thus it is enough to prove density. Let $\mathcal{N}_{0}$ be an arbitrary neighborhood of $f$. By Definition 4.2(3) there is $g_{0} \in \mathcal{N}_{0}$ such that $\varphi_{f} \prec \varphi_{g_{0}}$. If $\varphi_{f}$ is combinatorially equivalent to $\varphi_{g_{0}}$, Lemma 7.2 ensures that $\varphi_{f}$ is combinatorially stable. Otherwise, let $\mathcal{N}_{1} \subset \mathcal{N}_{0}$ be a neighborhood of $f$ where all maps are dominated by $g_{0}$. Such a neighborhood exists by Definition 2.1(1) and Definition 4.2(2). By Definition $4.2(3)$ there is $g_{1} \in \mathcal{N}_{1}$ such that $\varphi_{f} \prec \varphi_{g_{1}}$. If $\varphi_{g_{1}}$ is combinatorially equivalent to $\varphi_{g_{0}}$, Lemma 7.2 ensures that $\varphi_{g_{1}}$ is combinatorially stable. Otherwise we repeat the process considering a sequence of dominated maps

$$
\varphi_{f} \prec \cdots \prec \varphi_{g_{n}} \prec \cdots \prec \varphi_{g_{1}} \prec \varphi_{g_{0}} .
$$

It is a straightforward consequence of the definitions that we have:

$$
\left|\Lambda_{\text {final }}^{\Omega}\left(\varphi_{f}\right)\right| \geq\left|\Lambda_{\text {final }}^{\Omega}\left(\varphi_{g_{n}}\right)\right| \geq\left|\Lambda_{\text {final }}^{\Omega}\left(\varphi_{g_{n-1}}\right)\right| \geq\left|\Lambda_{\text {final }}^{\Omega}\left(\varphi_{g_{0}}\right)\right|
$$

and

$$
\left|\Sigma_{\text {final }}^{\Omega}\left(\varphi_{f}\right)\right| \geq\left|\Sigma_{\text {final }}^{\Omega}\left(\varphi_{g_{n}}\right)\right| \geq\left|\Sigma_{\text {final }}^{\Omega}\left(\varphi_{g_{n-1}}\right)\right| \geq\left|\Sigma_{\text {final }}^{\Omega}\left(\varphi_{g_{0}}\right)\right| .
$$

Furthermore, since each open map has a finite number of connected components, we must find an $n$ such that $\varphi_{g_{n}}$ is combinatorially equivalent to $\varphi_{g_{n-1}}$. By Lemma 7.2, $\varphi_{g_{n}}$ is combinatorially stable, which proves the Theorem.

\section{References}

[A] V. Araújo. Attractors and time averages for random maps. Ann. Inst. H. Poincaré Anal. Non Linéaire 17 (2000), 307-369.

[AF] J.-P. Aubin and H. Frankowska. Set-Valued Analysis. Birkhäuser, Boston, 2000 .

[B] P. Billingsley. Probability and Measure. Wiley Interscience, New York, 1985. 
[BDV] C. Bonatti, L. Díaz and M. Viana. Dynamics beyond uniform hyperbolicity: a global geometric and probabilistic approach. Encyclopaedia of Mathematical Sciences vol 102, Springer Verlag, 2004.

[Bo] R. Bowen. Equilibrium states and the ergodic theory of Anosov diffeomorphisms (Lecture Notes in Mathematics, 470). Springer, Berlin, 1975.

[BR] R. Bowen. and D. Ruelle. The ergodic theory of Axiom A flows. Invent. Math. 29 (1975), 181-202.

[D] J.L. Doob. Stochastic Processes. Wiley, New York, 1953.

[DT] P. Duarte and M. J. Torres. Combinatorial stability of non-deterministic systems. Ergodic Theory and Dynamical Systems 26 (2006), 93-128.

[K1] Y. Kifer. On small random perturbations of some smooth dynamical systems. Math. USSR Ivestija 8 (1974), 1083-1107.

[K2] Y. Kifer. Ergodic theory of random perturbations. Birkhauser, Boston Basel, 1986.

[K3] Y. Kifer. General random perturbations of hyperbolic and expanding transformations. J. Analyse Math 47 (1986), 11-150.

[K4] Y. Kifer. Random perturbations of dynamical systems. Birkhauser, Boston Basel, 1988.

[Li] P.-D. Liu. Dynamics of random transformations: smooth ergodic theory. Ergodic Theory and Dynamical Systems 21 (2001), 1279-1319.

[N] S. Nadler Jr. Hyperspaces of Sets (Pure and Applied Mathematics, A Series of Monographs and Textbooks). Marcel Dekker, New York, 1978.

[P] J. Palis. A global view of dynamics and a conjecture on the denseness of finitude of attractors. Astérisque 261 (2000), 335-347.

[R] W. Rudin. Functional Analysis. Tata McGraw-Hill Publishing Company LTD. New Delhi, 1982.

[Ru] D. Ruelle. A measure associated with Axiom A attractors. Amer. J. Math. 98 (1976), 619-654. 
[S] M. Shub. Global Stability of Dynamical Systems. Springer, New York, 1987.

[Si1] Ya. Sinai. Gibbs measure in ergodic theory. Russian Math. Surveys 27 (1972), 21-69.

[Si2] Ya. Sinai. Kolmogorov's work on ergodic theory. Ann. Prob. 17(3) (1989), 833-839.

[Sm] S. Smale. Differentiable dynamical systems. Bull. Am. Math. Soc. 73 (1967), 747-817.

[V1] M. Viana. Stochastic Dynamics of Deterministic Systems (Lecture Notes of the XXIst Brazil Mathematics Colloquium). IMPA, 1997.

[V2] M. Viana. Dynamics: a probabilistic and geometric perspective. Proceedings of the ICM, 1998. Documenta Mathematica I (1998), 557-578.

[W] L. Weis. Decompositions of positive operators and some of their applications. Functional Analysis: Surveys and Recent Results III. North-Holland (1983) 95-115.

[Y1] L.-S. Young. Stochastic stability of hyperbolic attractors. Ergodic Theory and Dynamical Systems 6 (1986), 311-319.

[Y2] L.-S. Young. What are SRB measures, and which dynamical systems have them? J. Statist. Phys. 108 (2002), 733-754. Dedicated to David Ruelle and Yasha Sinai on the occasion of their 65th birthdays. 